\title{
Understanding greater cardiomyocyte functions on aligned compared to random carbon nanofibers in PLGA
}

This article was published in the following Dove Press journal:

International Journal of Nanomedicine

17 December 2014

Number of times this article has been viewed

\author{
Abdullah M Asiri' \\ Hadi M Marwani' \\ Sher Bahadar Khan' \\ Thomas J Webster ${ }^{1,2}$ \\ 'Center of Excellence for Advanced \\ Materials Research, King Abdulaziz \\ University, Jeddah, Saudi Arabia; \\ ${ }^{2}$ Department of Chemical Engineering, \\ Northeastern University, Boston, MA, \\ USA
}

\begin{abstract}
Previous studies have demonstrated greater cardiomyocyte density on carbon nanofibers (CNFs) aligned (compared to randomly oriented) in poly(lactic-co-glycolic acid) (PLGA) composites. Although such studies demonstrated a closer mimicking of anisotropic electrical and mechanical properties for such aligned (compared to randomly oriented) CNFs in PLGA composites, the objective of the present in vitro study was to elucidate a deeper mechanistic understanding of how cardiomyocyte densities recognize such materials to respond more favorably. Results showed lower wettability (greater hydrophobicity) of CNFs embedded in PLGA compared to pure PLGA, thus providing evidence of selectively lower wettability in aligned CNF regions. Furthermore, the results correlated these changes in hydrophobicity with increased adsorption of fibronectin, laminin, and vitronectin (all proteins known to increase cardiomyocyte adhesion and functions) on CNFs in PLGA compared to pure PLGA, thus providing evidence of selective initial protein adsorption cues on such CNF regions to promote cardiomyocyte adhesion and growth. Lastly, results of the present in vitro study further confirmed increased cardiomyocyte functions by demonstrating greater expression of important cardiomyocyte biomarkers (such as Troponin-T, Connexin-43, and $\alpha$-sarcomeric actin) when CNFs were aligned compared to randomly oriented in PLGA. In summary, this study provided evidence that cardiomyocyte functions are improved on CNFs aligned in PLGA compared to randomly oriented in PLGA since CNFs are more hydrophobic than PLGA and attract the adsorption of key proteins (fibronectin, laminin, and vironectin) that are known to promote cardiomyocyte adhesion and expression of important cardiomyocyte functions. Thus, future studies should use this knowledge to further design improved CNF:PLGA composites for numerous cardiovascular applications.
\end{abstract}

Keywords: cardiomyocytes, poly(lactic-co-glycolic acid), carbon nanofibers, aligned, nanotechnology, anisotropy, mechanism, vitronectin, fibronectin, laminin

\section{Introduction}

Cardiovascular disease is a leading cause of death, with 785,000 cases of new heart attacks (or myocardial infarctions) and 470,000 cases of recurrent heart attacks reported in the US each year. ${ }^{1}$ Since a heart attack results in the large-scale loss of cardiac muscle, after a first heart attack, dead heart tissue remains and the subsequent weaker cardiac muscle may lead to additional myocardial infarctions. ${ }^{2-5}$ Because of the above, researchers have been developing numerous biomaterial strategies to regenerate healthy heart tissue in the place of the weakened tissue to alleviate the possibility of a subsequent heart attack. There has been considerable interest in developing injectable scaffolds (such as poly( $N$-isopropylacrylamide) [PNIPAAM], collagen, or helical rosette nanotubes), which offer the advantage of avoiding patient-specific scaffold
Correspondence: Thomas J Webster Department of Chemical Engineering, Northeastern University, Boston, MA 02115 , USA

$\mathrm{Tel}+\mathrm{I} 6173836585$

Fax +I 6173836586

Email th_webster@neu.edu 
prefabrication and highly invasive surgery. ${ }^{6}$ PNIPAAM has indeed improved cardiac functions in rabbit infarct models, preserving left ventricle ejection and preventing scar tissue formation, ${ }^{7,8}$ while collagen has thickened the infarcted wall and enhanced angiogenesis. ${ }^{9}{ }^{10}$ However, noninjectable cardiac patches also have their own distinct advantages, because with injectables, one has to be concerned with developing materials with quick solidification times to avoid permeation of the injectable throughout the body (which could cause toxic responses). Thus far, though, most injectable and noninjectable cardiac scaffolds developed by researchers have been nonconductive and non-anisotropic, such as those mentioned earlier, yet heart tissue is highly conductive and highly anisotropic (ranging from $0.16 \mathrm{~S} / \mathrm{m}$ [longitudinal] to $0.005 \mathrm{~S} / \mathrm{m}$ [transverse]). ${ }^{11}$
Along these lines, we previously reported that anisotropic cardiac patches composed of carbon nanofibers (CNFs) aligned in PLGA promoted cardiomyocyte density for up to 5 days in culture (Figure 1). ${ }^{12}$ Further, we demonstrated similar anisotropic mechanical and electrical properties of CNFs aligned in PLGA compared to CNFs randomly aligned in PLGA and pure PLGA, which may have led to significantly greater cardiomyocyte density on such composites. ${ }^{12}$ Specifically, the vertical (or longitudinal) conductivity increased from $0.075 \mathrm{~S} / \mathrm{m}$ to $0.1 \mathrm{~S} / \mathrm{m}$ and the transverse (or horizontal) conductivity decreased from $0.075 \mathrm{~S} / \mathrm{m}$ to $0.0025 \mathrm{~S} / \mathrm{m}$ for the CNFs randomly oriented compared to aligned in PLGA, respectively. ${ }^{12}$ Tensile tests also provided additional evidence of the superior mechanical properties of CNFs aligned in PLGA because previous results had

\section{A}

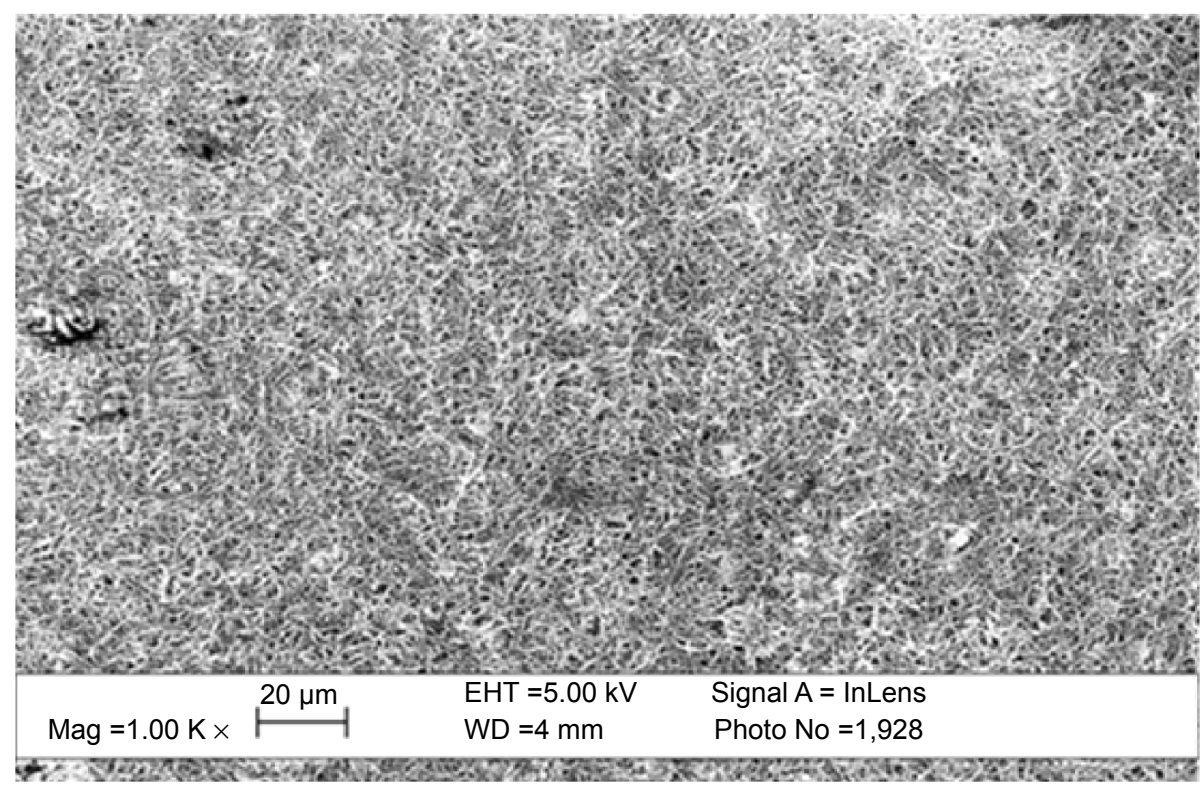

B

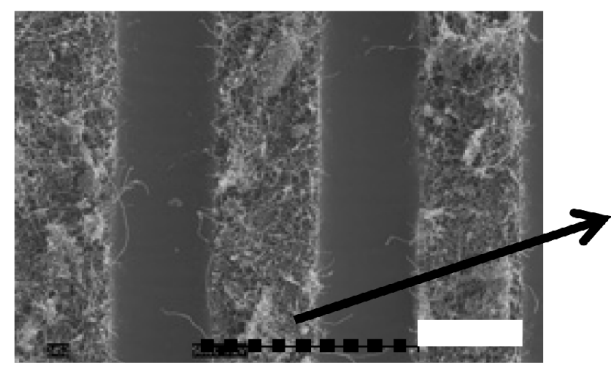

C

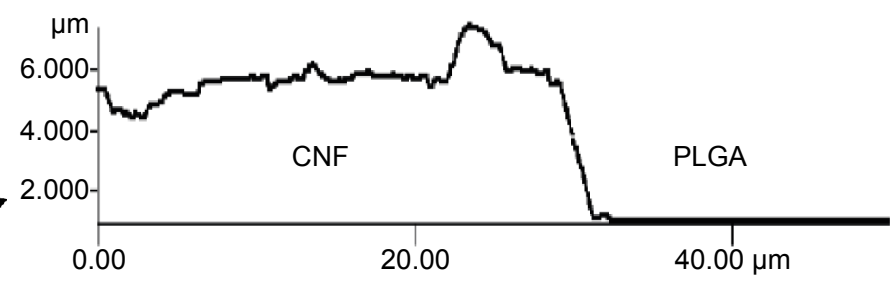

Figure I Previously fabricated CNFs aligned in PLGA that have shown greater cardiomyocyte density than both CNFs randomly oriented in PLGA and pure PLGA.

Notes: SEM images showing the distribution of CNF in the PLGA matrix at 50:50 wt\% at (A) random orientation and (B) aligned orientation to mimic the natural anisotropy of cardiac tissue. (C) AFM line scan of the 50:50 CNF:PLGA aligned composite, demonstrating micrometer-scale alignment of the CNFs on the PLGA surface. AFM scans for the randomly oriented CNFs in PLGA matched that of the CNF patterned region. Scale bars $=20 \mu \mathrm{m}$.

Abbreviations: AFM, atomic force microscopy; CNFs, carbon nanofibers; Cx43, Connexin-43; PLGA, poly(lactic-co-glycolic acid); $\alpha$-SCA, $\alpha$-sarcomeric actin; cTNT, Troponin-T. 
A

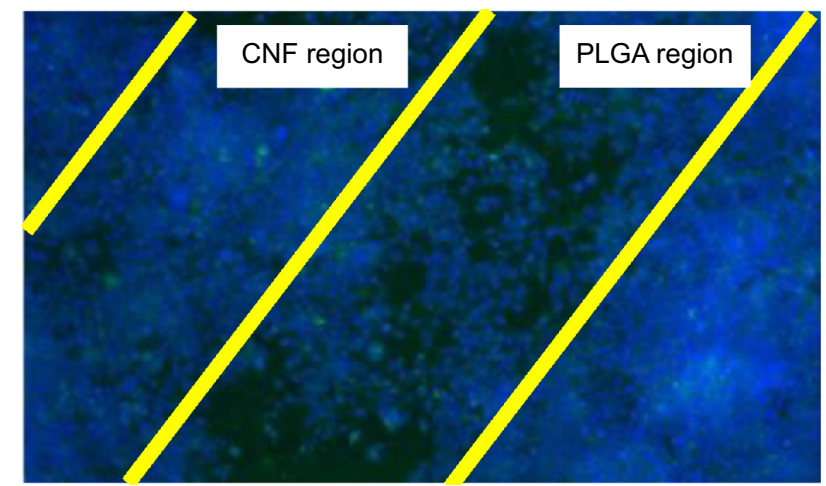

B

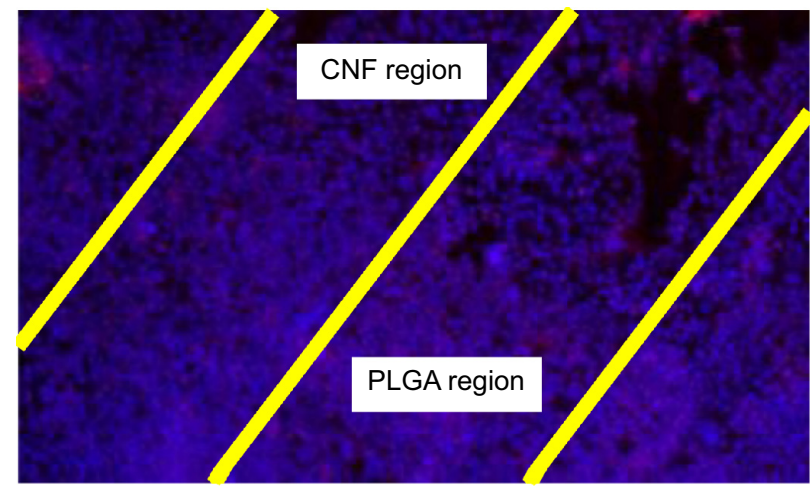

C

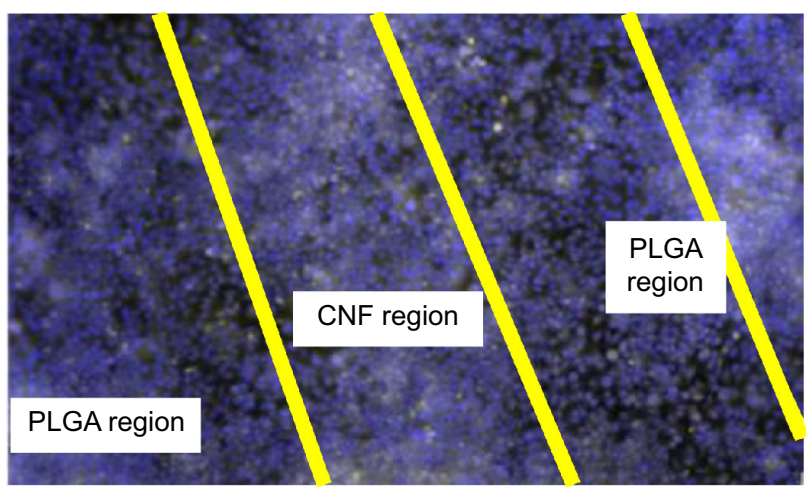

Figure 2 Fluorescent microscopy images of cardiomyocytes stained with various biomarkers on CNFs aligned on PLGA demonstrating significantly greater numbers of active cardiomyocytes on CNF compared to PLGA regions.

Notes: Each CNF and PLGA region is $200 \mu \mathrm{m}$ wide. Culture time $=120$ hours. (A) Representative image of cTNT (green) expression with DAPI (blue) stained cardiomyocytes on CNFs aligned on PLGA. (B) Representative image of Cx43 (red) expression with DAPI (blue) stained cardiomyocytes on CNFs aligned on PLGA. (C) Representative image of $\alpha$-SCA (yellow) expression with DAPI (blue) stained cardiomyocytes on CNFs aligned on PLGA.

Abbreviations: CNFs, carbon nanofibers; Cx43, connexin-43; DAPI, diamidino phenylindole dilactate; PLGA, poly(lactic-co-glycolic acid); $\alpha$-SCA, $\alpha$-sarcomeric actin; cTNT, Troponin-T.

shown that the Young's modulus was 5.05 MPa compared to $7.90 \mathrm{MPa}$ in the vertical direction and 4.96 MPa compared to $2.14 \mathrm{MPa}$ in the horizontal direction for the CNFs randomly oriented versus aligned in PLGA. ${ }^{12}$ Moreover, the elongation at break was $10.1 \%$ compared to $4.1 \%$ in the vertical direction and $11.3 \%$ compared to $14.8 \%$ in the horizontal direction for the CNFs randomly oriented versus aligned in PLGA. ${ }^{12}$ Compared to native cardiac heart tissue, the composites had better (stiffer) mechanical properties, with the former being elastomeric with a stiffness of approximately $50-100 \mathrm{kPa}$ during diastole. ${ }^{13-17}$ However, the modulus of active myocardium during systole is approximately 20 -fold higher, which more closely resembles the aligned CNF in PLGA composites. ${ }^{13-17}$

Despite the above promise shown by CNFs aligned in PLGA for cardiovascular applications, a fundamental understanding of greater cardiomyocyte densities on CNFs aligned in PLGA has not been elucidated to date. ${ }^{12}$ For these reasons, the objective of the present in vitro study was to determine the material properties of the CNFs aligned in PLGA that may be mechanistically responsible for the enhanced cardiomyocyte functions. In this paper, "functions" refers to the greater expression of proteins involved in cardiomyocyte functions (such as contractility, signaling, etc) and not those actual functions which will be the focus of future studies.

\section{Materials and methods PLGA:CNF fabrication}

The PLGA:CNF composites were fabricated as previously described. ${ }^{12}$ Since the 50:50 wt\% CNF in PLGA composites in our prior studies resulted in the best cardiomyocyte functions, this composition was selected here. ${ }^{18,19}$ Thus, PLGA composites (0.025 g/mL; 50:50 PLA:PGA wt \% [Polysciences, \#23986, USA]) were created by first diluting tetrahydrofuran (THF; Mallinckrodt Chemicals, \#C45763, USA) and sonicating the PLGA pellets in THF in a water bath (VWR B3500A-DTH, USA) below $30^{\circ} \mathrm{C}$ for 30 minutes. Five-hundred milligrams of CNFs $(99.9 \%$ by weight $\%$, Catalytic Materials, USA) with a diameter of $100 \mathrm{~nm}$ and $100 \mu \mathrm{m}$ long was sonicated (Misonix Sonicator 


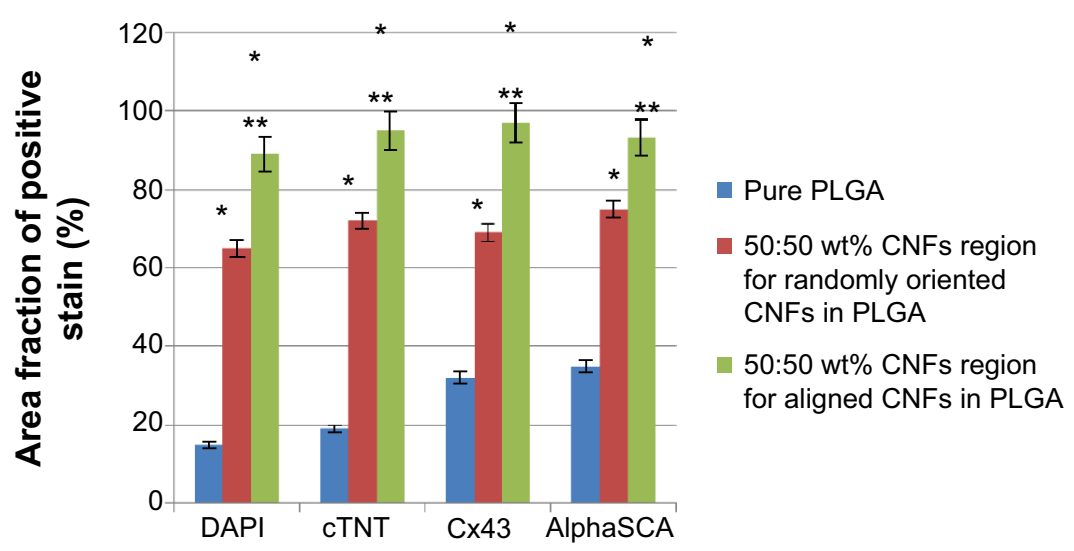

Figure 3 Increased area fraction (\%; the area of stain on the surface compared to the total area of the image) of active cardiomyocytes on CNFs aligned on PLGA samples. Data $=$ mean $\pm \mathrm{SEM} ; \mathrm{N}=3 ; * \mathrm{P}<0.0 \mathrm{I}$ compared to pure PLGA and $* * P<0.01$ compared to $50: 50$ wt $\%$ randomly oriented CNFs in PLGA. Culture time $=120$ hours.

Abbreviations: CNFs, carbon nanofibers; Cx43, connexin-43; DAPI, diamidino phenylindole dilactate; PLGA, poly(lactic-co-glycolic acid); $\alpha$-SCA, $\alpha$-sarcomeric actin; cTNT, Troponin-T.

3000, USA) in chloroform (Fisher Science, \#102591, USA) at $20 \mathrm{~W}$ for 30 minutes. After obtaining the separately sonicated PLGA and CNF solutions, PLGA:CNF solutions at $50: 50 \mathrm{wt} \%$ ratios were made by adding the appropriate amounts of CNF to PLGA. When the appropriate ratio of CNFs and PLGA was added, each composite material was sonicated (Misonix Sonciator 3000, USA) at $10 \mathrm{~W}$ for 20 minutes each.

Next, a 22-mm-diameter microscope cover glass (Fisher Scientific Circles \#1 [0.13-0.17 mm thick]; Size: $22 \mathrm{~mm}$, \#12-545-101, USA) was coated with the aforementioned composite. First, the glass substrate was cleaned by soaking in a 70:30 (vol\%) ethanol/deionized water solution while shaking (VWR, Advanced Digital Shaker, USA) for 10 minutes. Next, the substrate was immersed in $100 \%$ deionized water and shaken for 10 minutes. Using a disposable pipette (Fisherbrand, \#13-711-9AM, USA), $1 \mathrm{~mL}$ of the PLGA:CNF composite solution was placed on the glass substrate, which was then placed in an oven at $42^{\circ} \mathrm{C}$ for 15 minutes to cure. Each composite film was then vacuum-dried (Shel Lab, USA) at 20 in of $\mathrm{Hg}$ vacuum for 48 hours to allow the THF and chloroform to evaporate. Similar experiments were conducted for controls (without CNFs). All the samples were sterilized with ultraviolet light exposure for 24 hours prior to cell experiments.

To create aligned CNFs in PLGA, a similar process to that described above was followed, except that the PLGA:CNF composites in solution were exposed to $20 \mathrm{~V}$ after being placed on a coverslip and were then allowed to cure (which took approximately 24 hours). This resulted in the CNFs being aligned in the PLGA. Moreover, after solidification, micropatterns of CNFs were created on the PLGA surface by placing Au-coated grids with grooves (width $20 \mu \mathrm{m}$, catalog no 2422G-XA, SPI Supplies, West Chester, PA, USA) on top of the PLGA coating on the coverslips. The CNF-containing PLGA solution was then placed into the grooves of the $\mathrm{Au}$ grid by a micropipette. The grid with imbedded CNFs on top of the PLGA-coated glass was then placed in a vacuum oven for 48 hours, after which the Au grid was removed and the substrate rinsed with deionized water, thus creating microscale patterns of CNFs on the PLGA surface.

For the immunofluorescently stained samples (experiments to be described below), for the ease of experimentation, CNF aligned regions $200 \mathrm{~m}$ in diameter on PLGA were created.

\section{Material characterization}

\section{Contact angle tests}

As an estimation of wettability, contact angle measurements were made on the substrates of interest to the present study using a Krüss EasyDrop system (model DSA20S) with the Krüss static and dynamic contact angle software (SW4001). Drops for contact angle measurements were created by dropping $3 \mu \mathrm{L}$ of distilled water on the composites, and the angle between the surface and the water droplets was measured. Six measurements were made on each sample, which were further repeated thrice.

\section{Protein adsorption tests}

Because of the correlation between surface wettability and protein adsorption, protein adsorption on the samples of interest to the present study was evaluated using the wellestablished laminin, fibronectin, and vitronectin (all proteins known to mediate the adhesion of cardiomyocytes) enzymelinked immunosorbent assay (ELISA). All samples were 
rinsed with phosphate-buffered saline (PBS; solution containing $8 \mathrm{~g} \mathrm{NaCl}, 0.2 \mathrm{~g} \mathrm{KCl}, 1.5 \mathrm{~g} \mathrm{Na}_{2} \mathrm{HPO}_{4}$, and $0.2 \mathrm{~g} \mathrm{KH}_{2} \mathrm{PO}_{4}$ in $1,000 \mathrm{~mL}$ deionized water adjusted to a $\mathrm{pH}$ of 7.4; all chemicals from Sigma-Aldrich) three times. One milliliter of cardiomyocyte growth medium (Celprogen, \#M36044-15S, USA) with $10 \%$ fetal bovine serum (FBS, Thermo Scientific, \#SH30109, USA) was added to each well and incubated under standard conditions $\left(5 \% \mathrm{CO}_{2} / 95 \%\right.$ humidified air and $\left.37^{\circ} \mathrm{C}\right)$ for 1 hour. The medium was then aspirated, and the samples were rinsed twice with PBS. The samples were blocked by adding $500 \mu \mathrm{L}$ of a $2 \%$ bovine serum albumin (BSA) solution (BSA and PBS solution mix) for 1 hour onto the samples, which were then rinsed twice with PBS.

Five-hundred microliters of the first antibody (fibronectin [Chemicon, \#AB19014, USA]) containing solution (3 $\mu \mathrm{g}$ of the antibody in $500 \mu \mathrm{L}$ of a $1 \%$ BSA solution) was added to each sample, which was then incubated for 1 hour under standard conditions. After 1 hour, the antibody solution was aspirated and rinsed with $0.05 \%$ Tween $^{\circledR} 20$ (Sigma Aldrich, \#P1379-25ML, USA) solution three times while shaking (using a table shaker) for 5 minutes. After the last Tween ${ }^{\circledR}$ 20 aspiration, $500 \mu \mathrm{L}$ of a goat anti-rabbit secondary antibody conjugated with a horseradish peroxidase (Bio-Rad, \#170-6515, USA) solution (5 $\mu \mathrm{L}$ of the secondary antibody in $500 \mu \mathrm{L}$ in $1 \%$ BSA solution) was added to each well and incubated for 1 hour. Then, the secondary antibody was aspirated and rinsed three times with $0.05 \%$ Tween ${ }^{\circledR} 20$ while shaking (using a table shaker) for $5 \mathrm{~min}$. Five-hundred microliters of 2,2'-azino-bis(3-ethylbenzothiazoline-6-sulfonic acid) (ABTS) (Vector Labs Inc, Burling Game, CA, USA, \#SK-4500) was added to each well and incubated at room temperature in the dark for 20 minutes, while $200 \mu \mathrm{L}$ of each sample was read at $405 \mathrm{~nm}$ using a spectrophotometer. This was also performed for vitronectin and laminin (Chemicon International, Temecula, CA, USA; \#AB2047 and \#AB2068, respectively) as primary antibodies using the same process described above for fibronectin.

\section{Cardiomyocyte functions}

To correlate the above trends in wettability and initial adsorption of proteins important for cardiomyocyte functions on the substrates of interest to the present study, experiments with cardiomyocytes were also conducted. For this, cardiomyocytes were immunofluorescently stained for cardiomyocytespecific biomarkers: connexin-43 for gap junction channels (Cx43), $\alpha$-sarcomeric actin ( $\alpha$-SCA) for cardiac myocytes, and troponin $\mathrm{T}$ (cTNT) for contractile myocytes. ${ }^{20} \mathrm{Cx} 43$ is a protein present in the gap junctions between caromyocytes that mediate intercellular signaling and conduct electrical impulses. ${ }^{21} \alpha$-SCA is found only in cardiomyocyte- $\alpha$ skeletal and $\alpha$-cardiac muscle actin. ${ }^{22}$ Troponin T mediates cardiomyocyte contractibility as part of the troponin complex, which binds to thin myofilament actin.

Human cardiomyocytes (Celprogen, Torrance, CA, USA; \#36044-15, USA) were cultured in complete growth media (Celprogen, \#M36044-15S) supplemented with 10\% fetal bovine serum (Thermo Scientific, Waltham, MA, USA; \#SH30109) and 1\% penicillin/streptomycin (Fisher Scientific, Waltham, MA, USA; \#BP2959-50). Cells were seeded $\left(5 \times 10^{4}\right.$ cells $)$ onto the samples of interest to the present study in 12-well human cardiomyocyte stem cell culture extracellular matrix plates (Celprogen; \#E36044-15-12 Well) for 120 hours. The medium was then removed, and cell cultures were rinsed twice with PBS. Cells were fixed for 15 minutes with $1 \mathrm{~mL}$ of $10 \%$ formalin acetate (Fisher Scientific, \#SF99-20, USA) permeabilized with $0.2 \%$ Triton X-100 (Sigma-Aldrich, St Louis, MO, USA; \#X100-1L) diluted in PBS for 5 minutes, and blocked with an Image-iT FX signal enhancer (Invitrogen, Waltham, MA, USA; \#I36933) for 30 minutes at room temperature with two PBS wash steps in between each process. Primary rabbit polyclonal antiCx43 (Abcam, Cambridge, MA, USA; \#ab11370), mouse monoclonal anti-sarcomeric- $\alpha$ actin (Abcam, \#ab9465), and rabbit polyclonal anti-cardiac troponin T (Abcam, \#ab45932) antibodies were used at a dilution of 1:400 in PBS exposed to the cells for 1 hour at room temperature. Secondary AlexaFluor488 conjugated goat anti-mouse IgM (Invitrogen, \#A-21042) and anti-rabbit IgG (Invitrogen, \#A-21206) were diluted 1:600 in PBS and incubated with the cells for 60 minutes at room temperature. The nucleus was visualized using a diamidino phenylindole dilactate (DAPI) (Molecular Probes ${ }^{\circledR}$, Eugene, OR, USA; \#D1306) stain at a 1:30 dilution for 15 minutes at room temperature. Samples were then mounted with ProLong Gold Antifade (Invitrogen, \#P36930, USA) and imaged using a fluorescence microscope (Zeiss, Axiovert 200M, Berlin, Germany) with the ImageJ software (National Institutes of Health, USA) to quantify fluorescence. Images were converted to an 8-bit color scheme, then adjusted to a red/black threshold, and analyzed with the particle analysis tool to measure protein color concentration and compared to the total area of the image. This was done for all DAPI, cTNT, Cx43, and $\alpha$-SCA stains.

\section{Data and statistical analysis}

All experiments were performed in triplicate with three repeats each. Differences between means were determined 
using ANOVA followed by Student's $t$-test. A $P$-value of $<0.01$ was considered to be significant.

\section{Results}

\section{Material characterization}

Previously published results demonstrated aligned patterns of CNFs in PLGA when following the procedures mentioned above (Figure 1). ${ }^{12}$ For the randomly oriented CNFs in PLGA composites, CNFs were uniformly dispersed within the PLGA matrix (Figure 1A). However, as expected, for the aligned CNFs in PLGA, CNFs appeared in wellaligned $20 \mu \mathrm{m}$ patterns on PLGA, as further confirmed by the associated atomic force microscopy (AFM) line scan (Figure 1B and C). AFM scans for the randomly oriented CNFs in PLGA matched that of the CNF patterned region and, thus, are not shown.

\section{PLGA:CNF material properties Wettability}

Results of this study confirmed the different wettability properties between CNFs and PLGA, with CNFs demonstrating significantly greater hydrophobicity or lower surface energy than PLGA (Table 1). Thus, it can be expected that when creating linear patterns of CNFs in PLGA, different wettability properties were presented to alter the initial protein interactions and subsequent cardiomyocyte functions, as discussed next. It is fully expected that the slightly more hydrophobic proteins would adsorb on the more hydrophobic CNF regions than the less hydrophobic PLGA regions.

\section{Protein adsorption}

Results of this study correlated the decrease in surface energy for the CNFs compared to PLGA to the increased adsorption of fibronectin, laminin, and vitronectin after 1 hour (Table 2). While some studies have reported greater adsorption of these proteins on substrates with greater surface energy (ie, increased hydrophilicity), ${ }^{23}$ it appears that the surface

Table I Decreased wettability (increased hydrophobicity) of CNFs compared to PLGA

\begin{tabular}{ll}
\hline Sample & $\begin{array}{l}\text { Contact angle } \\
\text { (degrees) }\end{array}$ \\
\hline Pure PLGA & $82 \pm 3$ \\
$50: 50$ wt\% randomly oriented CNFs in PLGA & $135 \pm 2$ \\
$50: 50$ wt\% CNF region for aligned CNFs in PLGA & $145 \pm 3$ \\
\hline
\end{tabular}

Note: All values are significantly different from each other $(P<0.01)$.

Abbreviations: CNFs, carbon nanofibers; PLGA, poly(lactic-co-glycolic acid). energy of CNFs relative to PLGA provided a milieu appropriate for increasing the adsorption of fibronectin, laminin, and vitronectin.

\section{Cardiomyocyte functions}

Lastly, the results from this study demonstrated for the first time that the greater hydrophobicity of CNFs compared to PLGA, which promoted the adsorption of fibronectin, laminin, and vitronectin, led to increased cardiomyocyte functions. Specifically, after 120 hours of culture, greater numbers (as demonstrated by greater DAPI area fraction staining) were observed for CNFs aligned in PLGA compared to CNFs randomly oriented in PLGA, which were both greater than those in pure PLGA (Figures 2 and 3). Moreover, the cardiomyocytes were viable and active, as demonstrated by the greater positive staining for cTNT, $\mathrm{Cx} 43$, and $\alpha$-SCA. In fact, these specific markers for cardiomyocyte functions were greater when cultured on CNFs aligned in PLGA compared to CNFs randomly oriented in PLGA, which were both greater than those for pure PLGA after 120 hours (Figures 2 and 3). Lastly, results of this study showed greater normalized protein expression important for cardiomyocyte functions per cell number (determined using the aforementioned DAPI staining) on the aligned CNFs in PLGA compared to CNFs randomly oriented in PLGA, thus providing additional evidence of promoted cardiomyocyte functions on these novel substrates (Table 3).

\section{Discussion}

The myocardium is naturally conductive, contributing to the contraction of the heart as electrical signals are transmitted from the sinoatrial node to the atrioventricular node. After a heart attack, the conductivity of the myocardium is reduced as a result of cardiac tissue damage, which affects the transmission of signals and consequently contraction of the heart. Not surprisingly, because of this relationship between conductivity and the myocardium, Pedrotty et al showed that cardiac-like electrical stimulation increases myoblast proliferation. ${ }^{24}$ Despite this knowledge (known for centuries), few materials proposed to heal heart tissue damaged after a heart attack are conductive. In the present study, PLGA composites with CNFs also promoted cell numbers. Of course, a reason may be that the introduction of CNFs increased the conductivity of the PLGA composites closer to that of the heart tissue. ${ }^{12,18,19}$ Previous studies have also demonstrated that CNFs when added to PLGA improved mechanical properties to more closely match that of myocardial tissue. ${ }^{12,18,19}$ 
Table 2 Great adsorption of fibronectin, laminin, and vitronectin on CNFs compared to PLGA

\begin{tabular}{llll}
\hline Sample & $\begin{array}{l}\text { Fibronectin adsorption } \\
\text { intensity (arbitrary units) }\end{array}$ & $\begin{array}{l}\text { Laminin adsorption } \\
\text { intensity (arbitrary units) }\end{array}$ & $\begin{array}{l}\text { Vitronectin adsorption } \\
\text { intensity (arbitrary units) }\end{array}$ \\
\hline Pure PLGA & $0.65 \pm 0.02$ & $0.45 \pm 0.03$ & $0.25 \pm 0.03$ \\
50:50 wt\% randomly oriented CNFs in PLGA & $0.79 \pm 0.04$ & $0.58 \pm 0.02$ & $0.49 \pm 0.04$ \\
50:50 wt\% CNF region for aligned CNFs in PLGA & $0.92 \pm 0.1$ & $0.87 \pm 0.1$ & $0.71 \pm 0.05$ \\
\hline
\end{tabular}

Note: All values are significantly different from each other $(P<0.01)$.

Abbreviations: CNFs, carbon nanofibers; PLGA, poly(lactic-co-glycolic acid).

This study, thus, continues the promising work showing that CNFs could be added to nonconductive materials to impose conductivity (and mechanical) properties important for promoting cardiomyocyte functions. ${ }^{12,18,19}$ In addition to conductivity, CNFs can also mimic the dimensions of natural proteins, such as collagen, providing a more natural nanoscale roughness for myocardial tissue growth. Stout et al demonstrated that PLGA:CNF composites (when randomly oriented) promoted the growth of both cardiomyocytes and neurons. ${ }^{19} \mathrm{~A}$ potential problem with the use of CNFs in regenerative medicine, however, is their unknown toxicity. For example, while Kisin et al reported that CNFs induced genotoxicity when exposed to lungs ${ }^{25}$ no studies have investigated the toxicity of CNFs for myocardial applications. ${ }^{26}$ Clearly, such studies would have to be conducted before CNFs are further considered for cardiovascular applications.

However, one additional fact concerning the conductivity of heart tissue is that such conductivity is anisotropic, ranging from $0.16 \mathrm{~S} / \mathrm{m}$ longitudinally to $0.005 \mathrm{~S} / \mathrm{m}$ transversely. ${ }^{11}$ Thus, this study, for the first time, examined the influence of aligned CNFs versus randomly oriented CNFs in PLGA. In doing so, it was identified that, when emulating the anisotropic electrical properties of heart tissue by aligning CNFs in PLGA, further improvements in cardiomyocyte functions can result. Building on these exciting functions, an additional objective of this study was to determine the mechanism by which cardiomyocytes were improved on CNFs aligned in PLGA. To do this, we examined surface wettability and initial protein adsorption events important for mediating cardiomyocyte functions. Results of this study demonstrated that, in addition to simulating the electrical and mechanical properties of heart tissues, the CNF aligned regions in PLGA were more hydrophobic than PLGA to increase the adsorption of critical proteins that mediate cardiomyocyte functions, such as fibronectin, laminin, and vitronectin. Thus, in addition to biologically inspired electrical and mechanical properties, the ability of CNFs to increase the adsorption of fibronectin, laminin, and vitronectin is yet another reason for increased cardiomyocyte functions including cTNT, $\mathrm{Cx} 43$, and $\alpha$-SCA. The correlation between the surface energy of CNFs and further promoting the initial adsorption of these proteins should be the focus of future studies, which can continue to capitalize on such relationships to create even better cardiovascular tissue regenerative materials.

\section{Conclusion}

The objective of the present in vitro study was to provide a mechanistic understanding of why cardiomyocyte densities are greater on CNFs aligned in PLGA compared to randomly oriented CNFs in PLGA and pure PLGA. Results showed lower wettability (greater hydrophobicity) of CNFs embedded in PLGA compared to pure PLGA, thereby increasing the adsorption of fibronectin, laminin, and vitronectin (all proteins known to increase cardiomyocyte adhesion). Results of the present in vitro study further confirmed increased cardiomyocyte functions by demonstrating greater expression of important cardiomyocyte biomarkers (such as cTNT, Cx43, and $\alpha$-SCA) when cardiomyocytes were cultured on CNFs aligned in PLGA compared to randomly oriented in PLGA. In summary, this study provided evidence that cardiomyocyte functions are improved on CNFs aligned in PLGA compared to randomly oriented, since CNFs are more hydrophobic

Table 3 Greater normalized protein expression per cell on CNFs compared to PLGA

\begin{tabular}{|c|c|c|c|}
\hline Sample & $\begin{array}{l}\text { cTNT expression/cell } \\
\text { (arbitrary units) }\end{array}$ & $\begin{array}{l}\text { Cx43 expression/cell } \\
\text { (arbitrary units) }\end{array}$ & $\begin{array}{l}\alpha \text {-SCA expression/cell } \\
\text { (arbitrary units) }\end{array}$ \\
\hline Pure PLGA & $0.05 \pm 0.01$ & $0.15 \pm 0.04$ & $0.05 \pm 0.01$ \\
\hline 50:50 wt\% randomly oriented CNFs in PLGA & $0.13 \pm 0.02$ & $0.23 \pm 0.01$ & $0.20 \pm 0.03$ \\
\hline 50:50 wt\% CNF region for aligned CNFs in PLGA & $0.74 \pm 0.15$ & $0.67 \pm 0.11$ & $0.90 \pm 0.10$ \\
\hline
\end{tabular}

Notes: All values are significantly different than each other $(P<0.01)$. Determined from quantification of fluorescent stains.

Abbreviations: CNFs, carbon nanofibers; Cx43, Connexin-43; PLGA, poly(lactic-co-glycolic acid); $\alpha$-SCA, $\alpha$-sarcomeric actin; cTNT, Troponin-T. 
than PLGA and promote the adsorption of key proteins (fibronectin, laminin, and vitronectin), which, in turn, are known to promote cardiomyocyte adhesion and expression of important cardiomyocyte functions. Future studies should use this knowledge to further design improved CNF:PLGA composites for numerous cardiovascular applications.

\section{Acknowledgment}

This project was funded by the Deanship of Scientific Research (DSR), King Abdulaziz University, under grant no (25-130-35-HiCi). The authors acknowledge the technical and financial support of KAU.

\section{Disclosure}

The authors report no conflicts of interest in this work.

\section{References}

1. Lloyd-Jones D, Adams RJ, Brown TM, et al. Executive summary: heart disease and stroke statistics - 2010 update. Circulation. 2010;121: 948-954.

2. Jain AK, Knight C. Myocardial infarction - new definitions and implications. Medicine. 2010;38:421-423.

3. Laflamme MA, Murry CE. Regenerating the heart. Nat Biotechnol. 2005;23:845-856

4. Locca D, Bucciarelli-Ducci C, Ferrante G, et al. New universal definition of myocardial infarction: applicable after complex percutaneous coronary interventions? JACC Cardiovasc Interv. 2010;3:950-958.

5. Thygesen K, Alpert JS, White HD; on behalf of the Joint ESC/ACCF/ AHA/WHF Task Force for the Redefinition of Myocardial Infarction. Universal definition of myocardial infarction. $J$ Am Coll Cardiol. 2007;50:2173-2195.

6. Kretlow JD, Klouda L, Mikos AG. Injectable matrices and scaffolds for drug delivery in tissue engineering. Adv Drug Deliv Rev. 2007;59:263-273.

7. Wang T, Wu D-Q, Jiang X-J, et al. Novel thermosensitive hydrogel injection inhibits post-infarct ventricle remodelling. Eur J Heart Fail. 2009;11:14-19.

8. Li XY, Wang T, Jiang XJ, et al. Injectable hydrogel helps bone marrowderived mononuclear cells restore infarcted myocardium. Cardiology. 2010;115:194-199.

9. Dai W, Wold LE, Dow JS, Kloner RA. Thickening of the infarcted wall by collagen injection improves left ventricular function in rats: a novel approach to preserve cardiac function after myocardial infarction. $J \mathrm{Am}$ Coll Cardiol. 2005;46:714-719.

10. Huang NF, Yu J, Sievers R, Li S, Lee RJ. Injectable biopolymers enhance angiogenesis after myocardial infarction. Tissue Eng. 2005;11:1860-1866.
11. Roberts-Thomson KC, Kistler PM, Sanders P, et al. Fractionated atrial electrograms during sinus rhythm: relationship to age, voltage, and conduction velocity. Heart Rhythm. 2009;6:587-591.

12. Asiri AM, Marwani HM, Khan SB, Webster TJ. Greater cardiomyocyte density on aligned compared to random carbon nanofibers in polymer composites. Int J Nanomedicine. 2014;9(1):5533-5539.

13. Engelmayr GC, Cheng M, Bettinger CJ, Borenstein JT, Langer R, Freed LE. Accordion-like honeycombs for tissue engineering of cardiac anisotropy. Nat Mater. 2008;7(12):1003-1010.

14. Jalil J, Doering C, Janicki J, Pick R, Shroff S, Weber K. Fibrillar collagen and myocardial stiffness in the intact hypertrophied rat left ventricle. Circ Res. 1989;64(6):1041-1050.

15. Mirsky I, Parmley WW. Assessment of passive elastic stiffness for isolated heart muscle and the intact heart. Circ Res. 1973;33(2):233-243.

16. Boublik J, Park H, Radisic M, et al. Mechanical properties and remodeling of hybrid cardiac constructs made from heart cells, fibrin, and biodegradable, elastomeric knitted fabric. Tissue Eng. 2005;11(7-8):1122-1132.

17. Phillips CA, Petrofsky JS. Myocardial material mechanics: characteristic variation of the circumferential and longitudinal systolic moduli in left ventricular dysfunction. J Biomech. 1984;17(8):561-568.

18. Stout DA, Basu B, Webster TJ. Poly(lactic-co-glycolic acid): carbon nanofiber composites for myocardial tissue engineering applications. Acta Biomater. 2011;7(8):3101-3112.

19. Stout DA, Yoo J, Santiago-Miranda AN, Webster TJ. Mechanisms of greater cardiomyocyte functions on conductive nanoengineered composites for cardiovascular applications. Int J Nanomedicine. 2012;7:5653-5669.

20. Zheng H-x, Liu S-s, Tian W-m, Yan H-j, Zhang Y, Li Y. A threedimensional in vitro culture model for primary neonatal rat ventricular myocytes. Curr Appl Phys. 2012;12(3):826-833.

21. Tribulova N, Shneyvays V, Mamedova LK, et al. Enhanced connexin-43 and $\alpha$-sarcomeric actin expression in cultured heart myocytes exposed to triiodo-1-thyronine. J Mol Histol. 2004;35(5):463-470.

22. Carrier L, Boheler KR, Chassagne C, et al. Expression of the sarcomeric actin isogenes in the rat heart with development and senescence. Circ Res. 1992;70(5):999-1005.

23. Khang D, Kim SY, Liu-Synder P, Palmore GTR, Durbin SM, Webster TJ. Enhanced fibronectin adsorption on carbon nanotubes/poly(carbonate) urethane: independent role of surface nano roughness and associated surface energy. Biomaterials. 2007;28(32):4745-4768.

24. Pedrotty DM, Koh J, Davis BH, Taylor DA, Wolf P, Niklason LE. Engineering skeletal myoblasts: roles of three-dimensional culture and electrical stimulation. Am J Physiol Heart Circ Physiol. 2005;288:H1620-H1626.

25. Kisin ER, Murray AR, Sargent L, et al. Genotoxicity of carbon nanofibers: are they potentially more or less dangerous than carbon nanotubes or asbestos? Toxicol Appl Pharmacol. 2011;252(1):1-10.

26. Christman KL, Lee RJ. Biomaterials for the treatment of myocardial infarction. J Am Coll Cardiol. 2006;48(5):907-913.
International Journal of Nanomedicine

\section{Publish your work in this journal}

The International Journal of Nanomedicine is an international, peerreviewed journal focusing on the application of nanotechnology in diagnostics, therapeutics, and drug delivery systems throughout the biomedical field. This journal is indexed on PubMed Central, MedLine, CAS, SciSearch $\AA$, Current Contents $₫ /$ Clinical Medicine,

\section{Dovepress}

Journal Citation Reports/Science Edition, EMBase, Scopus and the Elsevier Bibliographic databases. The manuscript management system is completely online and includes a very quick and fair peer-review system, which is all easy to use. Visit http://www.dovepress.com/ testimonials.php to read real quotes from published authors. 